\title{
Evaluation of OpenFlow Load Balancing for Navy
}

\author{
Raheleh Dilmaghani, Dae Kwon \\ SPAWAR Systems Center Pacific \\ San Diego, CA \\ raheleh.dilmaghani, dae.kwon@navy.mil
}

\begin{abstract}
Load balancing is critical in complex naval networks to provide reliable performance by ensuring the system is not overloaded and by high resource utilization. Load balancing guarantees the stability of a naval ship tactical network in a distributed environment. Tactical networks are highly dynamic due to mission requirements, policies, or environment condition. There is never enough bandwidth for dataintensive military operations. Additional challenges of tactical environments are limited bandwidth, and intermittent connectivity caused by battle space condition. In this paper, we present our approach to create a benchmark to evaluate load balancing with OpenFlow protocol of SDN technology for a Navy scenario. Our results show that SDN technology offers agility and flexibility to configure, and manage the network.
\end{abstract}

\section{Keywords}

Software-Defined networking, OpenFlow, Load balancing

\section{INTRODUCTION}

Open Flow is the Open Networking Foundation's (ONF) standard on control protocol of Software-Defined Networking (SDN) [1].

SDN makes network programmable by separating the data and the control plane where the data plane is responsible in forwarding the traffic, and the control plane determines where the data should be forwarded to. SDN relies on switches that can be programmed through an SDN controller using a control protocol. The idea of open, programmable networks that enable agility and flexibility, has certainly resonated with navy, and throughout DoD. Programmable networks seem to mitigate challenges facing the network in providing state-of-art infrastructure to keep pace with expanding application demands and to keep cost, complexity, and labor needs under control.
OpenFlow is the software package that takes forwarding decisions based on rules defined by network administrator. A new packet of a flow is forwarded to the controller by the switch to find out where it should be routed to. Commands are translated into low-level instructions for data plane and appears as a flow entry and the rest of flow follows the entry in forwarding table. This work presents our approach to create a benchmark for experimentation and the results on evaluating load balancing capabilities of OpenFlow protocol in a navy environment.

Organization of the rest of the paper is as following: Section 2 presents a summary of related work on OpenFlow and software defined networking. Section 3 presents characteristics and requirements of tactical environments. Section 4 describes the scenario and experimentation configuration. Section 5 presents results of load balancing in our navy scenario followed by a summary in Section 6 .

\section{RELATED WORK}

In [2], the authors present the results of their survey on implementation, testing and applications of SDN. They present two different SDN architecture including ForCES [3] and OpenFlow describing their differences in terms of design, architecture, forwarding model, and protocol interface. In [4], the differences of the two architecture are presented for enterprise networks, data center, optical networks, home and small business, infrastructure-based wireless access network such as cellular and Wi-Fi where the last one mostly focuses on approaches and recommendation on provided vision for subsequent work. In [5], authors present their results from studying OpenFlow with different tools and its load balancing capabilities. In [6], the author suggest separating intelligence from data path and its potential benefits compared to traditional networks with built-in functions within the infrastructure.

In [7], the authors address challenges of network performance, scalability, security, and interoperability concerning wide use of cloud services and virtualization of data centers in order to take advantage of the predictability, continuity, and quality of service delivered by virtualization technologies. In [8], the authors present use cases for Defense community. In [9] and [10], the authors present a comprehensive survey on SDN and design issues. In [11], the author presents approaches and use cases of SDN. In [12], the authors present a use-case specific analysis to evaluate network management functionality of SDN. 


\section{TACTICAL ENVIRONMENT}

Tactical environments include different entities communicating over multiple available network technologies; however as the environment condition or mission requirements change, link availability, bandwidth or access policies need to be adapted accordingly; The members of coalition group may change which impacts the routing and services in the network. In real-life scenarios, often a network engineer needs to fly to an off-site location just to fix configuration of a box which is costly.

The design and fielding of an agile and secure tactical network is a key requirement. The ability to send right information to the right entities at the right time over common infrastructure is critical. Current network infrastructure provides security by static physically separated networks. However use of statically or physically separated network places a heavy burden on the infrastructure by requiring redundant, often identical hardware. Additionally it places a heavy burden on network configuration. Due to limitation in Size, Weight and Power (SWaP) in tactical and mobile platforms, operational capabilities may be compromised. Achieving security via physically separated networks also reduce network agility. Considering multiple domains of security even worsens the problem. With automating configuration, troubleshooting is reduced since most of errors are human-made configuration are removed. A software approach seems to lend itself to tactical environment as it can provide dynamic services and updates to the routing remotely rather than individual hardware.

Considering tactical operation's need for continuous and reliable service, raises the need for programmable network controller in a distributed environment. SDN can improve dynamic network management and security with the central controller with the high-level view of the network. Policy rules can easily and quickly be modified from a controller with central view which mitigates dynamic traffic and bandwidth management by updating flow control rules. One single controller is subject to single point of failure. A centralized management miles away from coalition forces in an operational scenario is challenging. The number and placement of controllers is an ongoing research problem that will be addressed in our future work. However the controller needs to be highly available and secure to ensure continuous support for network management. Logging of all traffic going through and individual filtering technologies are among other special requirements.

\section{EXPERIMENTATION}

In this section, we describe the scenario and the experimentation configuration used to create the load balancing benchmark for navy scenario. In Navy environment, in order for a ship to talk to another ship, the communication goes over satellite to shore, to Navy Operations Center (NOC), and then from shore to ship.

\subsection{Experimentation Setup}

We have created the network topology for this work using Mininet [13] on a virtual machine. Mininet is a virtual environment that its performance is limited to the resource of the host machine. We have evaluated different controller protocols and selected Floodlight as the OpenFlow protocol for the communication between controller and switches. During our experimentation, we observed that the Floodlight GUI is not reliable and is not able to update correctly.

Figure 1 shows the topology of the network under experimentation. The network topology consists of 5 Wide Area Network (WAN) switches, 3 ships and 2 data centers at shore, in which large volumes of data and voice are being transferred over WAN links. The WAN switches are interconnected to each other as shown in the diagram. There may be more than one satellite connection available to a ship at a point of time. In this scenario, each link has mul-

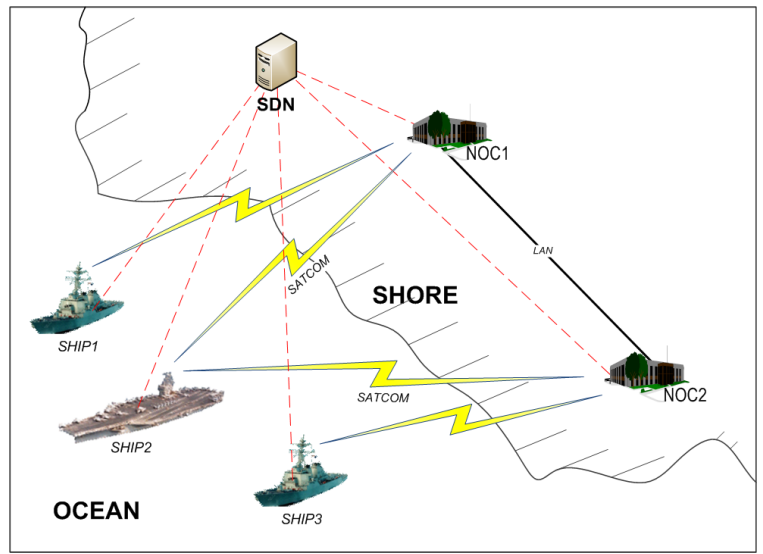

Figure 1: Experiment Network Topology

tiple queues to transmit data traffic. Each queue has been configured to handle data traffic for a given bandwidth. We assume three types of service requirement for three different types of data. Packets are generated with a Differentiated Services Code Point (DSCP) to distinguish different types of traffic to provide QoS and we observe and verify the corresponding Type of Service (ToS) when capturing the packet. Table 1 shows configuration between switches and hosts. Table 2 shows the connection setup, data rate and delay for the links. Switches provide QoS with simple queuing mechanism. A port can host multiple queues.

We assume 3 types of traffic: Type 1 indicates the highest priority and Type 3 indicates the lowest priority.

Type 1 - DSCP : 26, TOS : 104(0X68)

Type $2-D S C P: 18, T O S: 72(0 X 48)$

Type $3-D S C P: 10, T O S: 40(0 X 28)$

The controller injects flow entries to each WAN switch so that QoS for the traffic is provided by forwarding the traffic to the appropriate WAN switch. The controller is monitoring the WAN switches and links for any topology change in the network. The controller is programmed such that it can update flow entries depending on the environment or mission condition. In this experimentation, the queues are defined for each OpenFlow Vswitch as appears in Table 3. Figure 2 shows the network configuration for switches and hosts.

\subsection{Scenario Description}

The commanding officer of a ship receives a new mission package that requires changes in configuration and security access of the tactical network. The critical data needs to be 
Table 1: Switches and Host Configuration

\begin{tabular}{|l|l|l|l|l|}
\hline Number & Switch & Gateway & host number & host IP address \\
\hline 1 & $S W 1$ & Ship 1 & host1 & $10.0 .0 .1 / 24$ \\
\hline 2 & $S W 2$ & NOC 1 & host2 & $10.0 .0 .2 / 24$ \\
\hline 3 & $S W 3$ & NOC 2 & host3 & $10.0 .0 .3 / 24$ \\
\hline 4 & $S W 4$ & Ship 2 & host4 & $10.0 .0 .4 / 24$ \\
\hline 5 & $S W 5$ & Ship 3 & host5 & $10.0 .0 .5 / 24$ \\
\hline
\end{tabular}

Table 2: Links Setup

\begin{tabular}{|l|l|l|l|l|}
\hline Number & Between & Type & Rate & Round-Trip Delay (Sec) \\
\hline 1 & $S W 1-S W 2$ & SATCOM & $10 M b p s$ & 1 \\
\hline 2 & $S W 2-S W 3$ & LAN & $100 M b p s$ & 0 \\
\hline 3 & $S W 2-S W 4$ & SATCOM & $5 M b p s$ & 1 \\
\hline 4 & $S W 3-S W 4$ & SATCOM & $20 M b p s$ & 1 \\
\hline 5 & $S W 3-S W 5$ & SATCOM & $10 M b p s$ & 1 \\
\hline
\end{tabular}

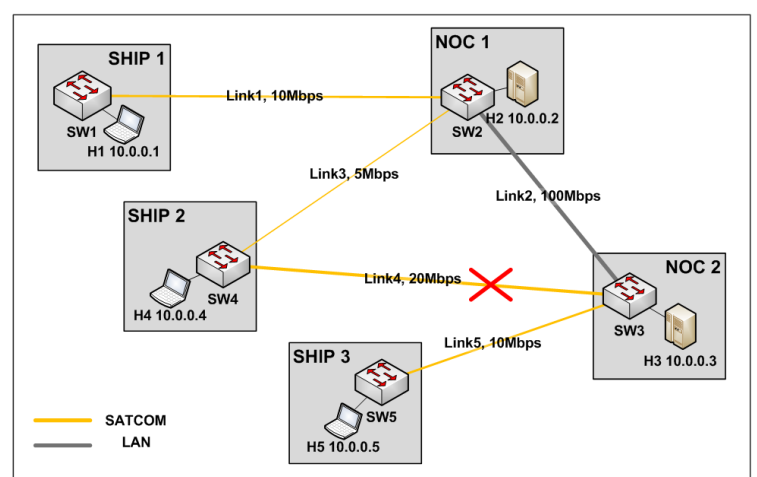

Figure 2: Network Configuration

sent to the members of operation. The data will travel from ship to shore, going through one or more Navy Operation Center (NOC) before reaching the destination, i.e. another ship. As the environmental conditions change, there is connectivity disruption on available paths. Similarly, access policy rules change based on mission requirements which may lead to an entity or a route to be excluded from the communication path or added, which in turn is translated to updates to flow rules. Depending on condition, the link availability or available bandwidth changes and load balancing service is required to adapt to the new condition rapidly to ensure reliable and agile performance. SDN controller and programmable interface provides dynamic adaptability as traffic requirements, rules, or the topology changes. During the mission, a direct SATCOM link between two nodes, Ship 2 and NOC 2 drops out. SDN controller learns about the disconnection and quickly adapts by updating flow entries according to the policies so that traffic is re-routed through alternative available links to reach the destination. In this scenario, we verify that the flow entries are correctly implemented and reshape the traffic according to the required QoS profile to accommodate to the lower bandwidth available.

In the second part of experiment, the dropped link is re- stored within a certain time. The SDN controller simply restores the original flow rules back to the switches so that the network continues to route traffic exactly as prior to the link unavailability. With the programmability of the controller, the flow rules can easily be reconfigured to route traffic or take different actions, depending on the circumstances or policy rules. Flow rules enable the network to distribute the current load of data traffic to balance over distinctive available paths or alternate full utilization. In this scenario, we define and verify that flow entries are correctly implemented to reroute the traffic for load balancing with bandwidth and reliability constraints.

\subsection{Controller Setup}

Upon startup, the SDN controller establishes connection to all switches and pushes default flow entries out to switches. In this experimentation, we assume SATCOM link (Link4) between the Ship2(SW4) and $N O C 2(S W 3)$ fails due to environment conditions. Switches $S W 3$ and $S W 4$ both send notification immediately to the controller. The controller re-routes data traffic through other node to reach the destination. The controller removes and adds flow entries on both $S W 3$ and $S W 4$. New data traffics are being relayed through the NOC1(SW2). The new path has much less bandwidth availability than the previous20Mbps. Therefore, the link needs to adapt much stricter QOS policy to accommodate the smaller bandwidth availability. The SDN controller pushes new flow rules to meet QOS policies to switches.

The SDN controller continues to monitor the network and manages the network when formerly dropped SATCOM link restores its connection. Ship 2 has greater bandwidth capability to NOC2. Based on demand of each traffic types, load balancing and sharing is feasible between two available links. The flow rules can be configured to transfer all traffic over restored link immediately or gradually depending on the policies.

\section{RESULTS}

Packets are generated and being sent from host 4 to host 3 through physical ports of the switches. Figure 3 shows the 
Table 3: Queue Setup

\begin{tabular}{|l|l|l|l|l|l|}
\hline Queue Number & Rate & Queue Number & Rate & Queue Number & Rate \\
\hline$Q 0$ & $50 M b p s$ & $Q 4$ & $6 M b p s$ & $Q 8$ & $2 M b p s$ \\
\hline$Q 1$ & $30 M b p s$ & $Q 5$ & $5 M b p s$ & $Q 9$ & $1 M b p s$ \\
\hline$Q 2$ & $20 M b p s$ & $Q 6$ & $4 M b p s$ & $Q 10$ & $0 M b p s$ \\
\hline$Q 3$ & $10 M b p s$ & $Q 7$ & $3 M b p s$ & - & - \\
\hline
\end{tabular}

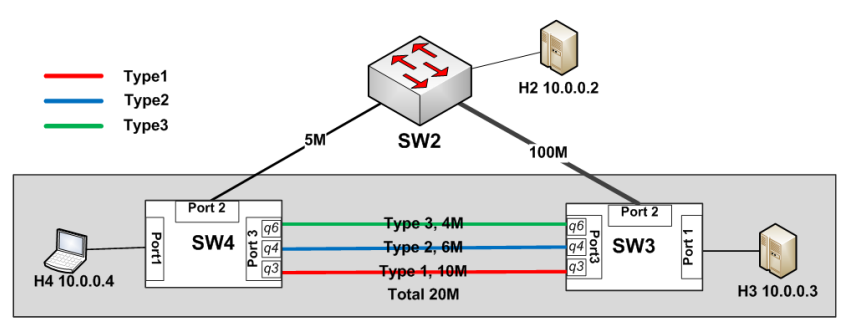

Figure 3: Physical Path and ToS between Hosts 3 and 4

path and settings between the two hosts.

host $4<-->$ sw 4 : port $1<-->$ sw 4 : port $3<-->$ sw $3:$ port $3<-->$ sw $3:$ port $1<-->$ host 3

Upon initiation, according to the flow rules, flow entries are populated in the corresponding switch as shown in Figure 4. The flows uploaded on $S W 4$ are defined to allow traffic to flow. Notice the packet counter increments as a flow matches with incoming packet. Five ping packets are generated from host4(10.0.0.4) to host3(10.0.0.3). These ping packets are not associated with any TOS(DSCP) marking and hence they are not going through a queue according to the defined action. Figure 5 shows IPERF test for QOS TYPE- 1 packets marked as TOS $=104(0 X 68)$. Packets are transmitted from host 4 to host3. Notice the maximum bandwidth for QOS TYPE1 is configured for 10Mbps and the tested result is 6.67Mbps. Again, the outbound flow entry in the $S W 4$ shows the matching packets for the TYPE1 traffic.

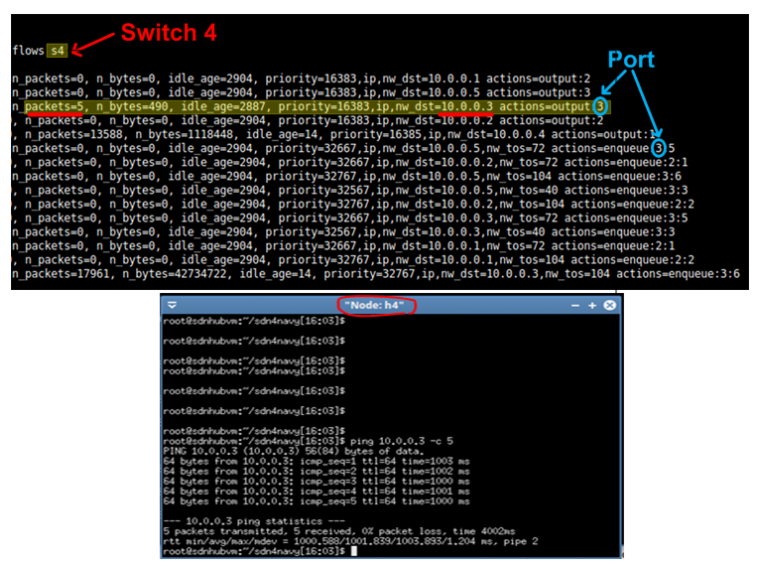

Figure 4: Flow Entries and Ping Results for $S W 4$

When the link between $S W 4$ and $S W 3$ drops, the SDN controller reconfigures network so that packets are sent out over other available link. Available bandwidth between $S W 4$ and $S W 2$ is $5 M b p s$ which is significantly less than the dropped

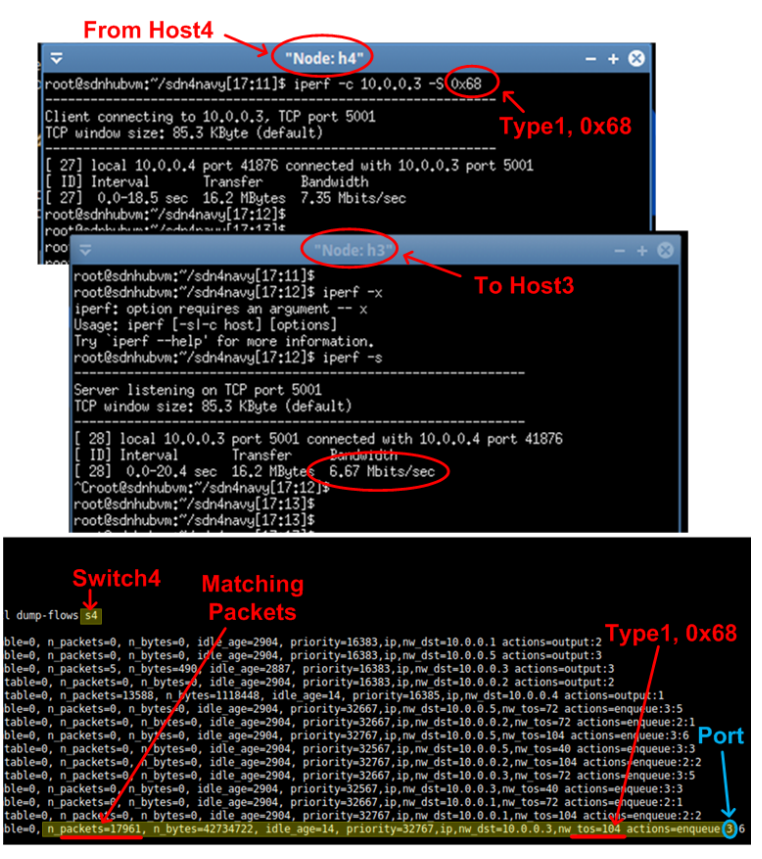

Figure 5: flows for TOS 104(0x68) Traffic for $S W 4$

link of 20Mbps. Mission planner can reconfigure the network so that one or more TOS traffic can be throttled down to enable the network to allow certain high priority traffic to be sent. In this example, the Ship $2(S W 4)$ allows only Type 1 and Type 2 traffics to go out to the $N O C 1(S W 2)$ and discards low priority Type 3 traffics as shown in Figure 6 . Packets are now being sent from the host 4 to host 3 through different physical ports of the switches as shown in Figure 7. host $4<-->$ sw 4 : port $1<-->$ sw 4 : port $2<-->$ sw $2:$ port $4<-->$ sw $2:$ port $3<-->$ sw $3:$ port $2<$ $-->$ sw $3:$ port $1<-->$ host 3

Next, new flows are pushed to the switches $S W 4$ and $S W 3$ to re-route the packets through SW2. SW4 drops TOS Type 3 traffic and allows only Type 1 and Type 2 traffic to pass. Switch $S W 3$ no longer sends traffic out to port3 since the link is down. It re-routes traffic out to port2 where $S W 2$ is connected.

Figure 8 shows Internet Performance (IPERF) results for QoS Type $1(0 X 68)$ and Type $2(0 X 48)$ packets. They are transmitted simultaneously from host 4 to host3. Notice the new bandwidth between Ship2(host4) and NOC2(host 3 ) is the maximum of $5 \mathrm{Mbps}$ because traffic are being relayed over the $N O C 1(S W 2)$. In the second part of scenario, the formerly disconnected 20Mbps SATCOM link becomes available again, the controller can allow the lowest priority 


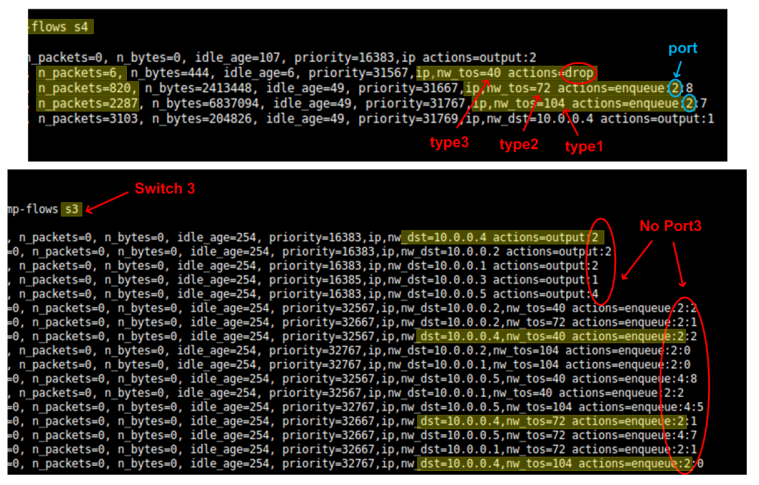

Figure 6: Flows in SW4 and SW3 after link down

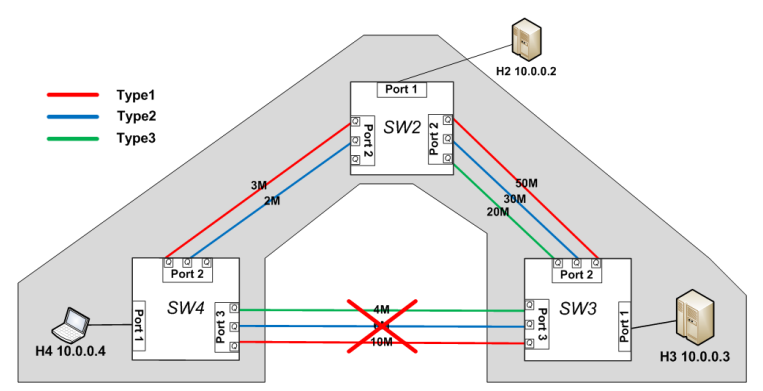

Figure 7: Updated TOS setup between Host4 and Host3

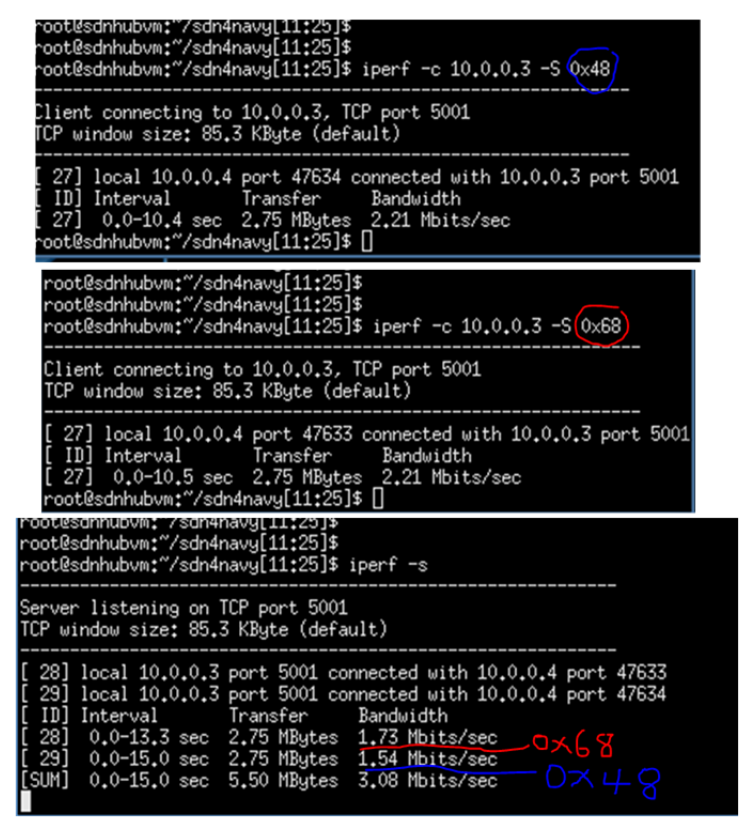

Figure 8: Data Performance for TOS Type1 and Type2 traffic Type 3 to transmit from Ship2(SW4). The link connection is restored but it can be unstable and shaky at the beginning. The controller continues to monitor the link and once the link becomes stable, other higher priority traffic can be transmitted over the link again. Upon link recovery, only Type 3 traffic and some Type 2 are being sent over the new link. Type 1 and most of Type 2 traffic are continued to be sent over previous $5 \mathrm{Mbps}$ link until the new link is proven to be reliable. The user can write a set of rules to define how the reliability of the link is determined. Also, rules contain types and amount of traffic to be transmitted while the link is being under evaluation for reliability. In this experimentation, we consider a link unreliable if it drops 3 or more times after link has recovered within 3 minute time window.

Figure 9 shows the newly recovered path. Type 3 and some of Type 2 traffic are now being sent from the host 4 to host 3 through the link. In Figure 10, flow entries are added in

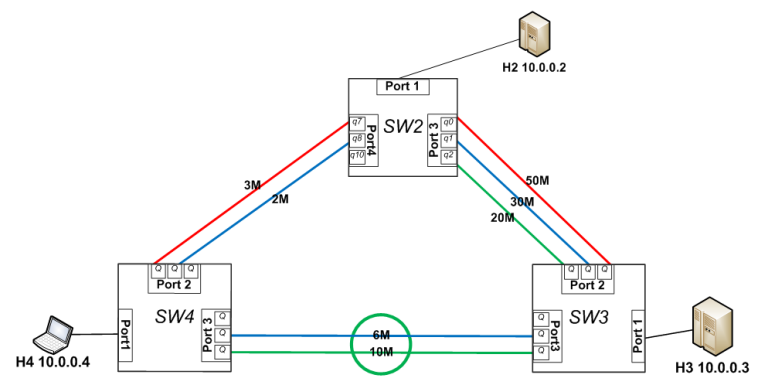

Figure 9: Temporary TOS setup between Host4 and Host3

Switch 4 to pass Type 3 traffic and Type 2 traffic that are destine to Host 3 and Host 5 are also transmitted over the new link which uses Port3. However, Type 2 traffic with destination to Host1 and Host2 continues to be transmitted over the same $5 \mathrm{Mbps}$ link. This allows load balancing of Type 2 traffic over two distinctive links. After a pre-defined time

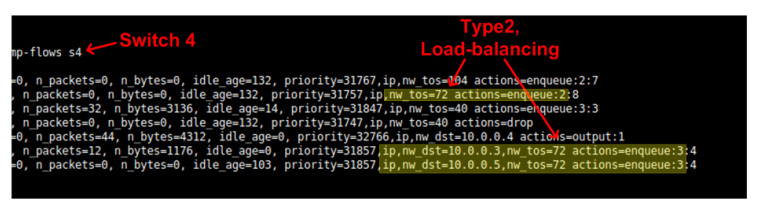

Figure 10: Temporary flow entries for $S W 4$

has elapsed, the recovered link is determined to be reliable, and former flow rules are reconfigured on $S W 4$ so that all traffic types are transmitted over the link as it was initially set as shown in Figure 11. To the best of our knowledge, we are not aware of Mininet Open vSwitch capability to provide analysis on packet lost or live bandwidth availability. Hence, the controller cannot depend on the switches to provide link quality matrix other than the link existence. TCLink is the interface in Mininet customized by Traffic Control (TC) to define the specifications of the link. Open VSwitch do not apply QoS settings to a port if the port is configured to use TCLink parameters such as bandwidth, delay, or queuemax-size. 


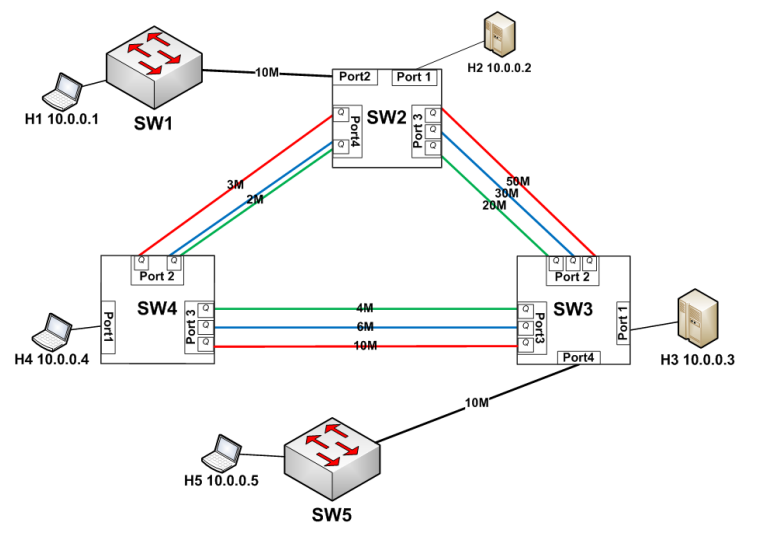

Figure 11: TOS setup of switches

Existing network devices are capable of achieving similar functionality but reconfiguration needs to be done per box. Also, since the control is located on the actual box, it can be exposed to various attacks. Placing controller in a secure place can increase the security of network management. Manually configuring each network device and service for every application is error-prone and time consuming. SDN enables the administration to configure the network and provision resources in much less time and more efficiently.

\section{CONCLUSION}

In this work, we implemented and evaluated the feasibility and advantages of a programmable network with FloodLight implementation of OpenFlow protocol in a navy scenario in a ship to shore scenario. SDN offers network automation that enables dynamic policy-based bandwidth and traffic management.

We verified how a central controller can adapt to the changes in the network; This reduces troubleshooting significantly since most errors in today network are caused by human error. Programmable network facilitates load balancing by ensuring reliability and resource utilization. We have identified challenges of implementation and graphics interface using the existing tools.

Availability of Network Application Interfaces (APIs) can be expanded to add on capabilities of OpenFlow. Additional developments can be implemented to extend the usability of the protocol in DIL environments.

\section{Acknowledgment}

This research acknowledges support as a Science and Technology Initiative Project under the Business Portfolio Council, SPAWAR Systems Center Pacific.

\section{REFERENCES}

[1] N. McKeown, T. Anderson, H. Balakrishnan, G. Parulkar, L. Peterson, J. Rexford, S. Shenker, and J. Turner. Openflow: enabling innovation in campus networks. ACM SIGCOMM Computer Communication Review, 38(2):69-74, 2008.

[2] B. Nunes, M. Mendonca, X. Nguyen, K. Obraczka, and T. Turletti. A survey of software-defined networking: Past, present, and future of programmable networks. 2014.

[3] A Doria, J H. Salim, R. Haas, H. Khosravi, W. Wang, L. Dong, R. Gopal, and J. Halpern. Forwarding and control element separation (forces) protocol specification. Internet Requests for Comments, RFC Editor, RFC, 5810, 2010.

[4] T. Tsou, X. Shi, J. Huang, Z. Wang, and X. Yin. Analysis of comparisons between openflow and forces. Analysis, 2012.

[5] S. Govindraj, A. Jayaraman, N. Khanna, and K. R. Prakash. Openflow: Load balancing in enterprise networks using floodlight controller. University of Colorado, 2012.

[6] N. McKeown. Software-defined networking. INFOCOM keynote talk, 17(2):30-32, 2009.

[7] S. Sezer, S. Scott-Hayward, P-K Chouhan, B. Fraser, D. Lake, J. Finnegan, N. Viljoen, M. Miller, and N. Rao. Are we ready for sdn? implementation challenges for software-defined networks. Communications Magazine, IEEE, 51(7):36-43, 2013.

[8] M. Mitchiner and R. Prasad. Software-defined networking and network programmability: Use cases for defense and intelligence communities. Cisco White Paper, 2014.

[9] D. Kreutz, F. Ramos, and P. Verissimo. Towards secure and dependable software-defined networks. In Proceedings of the second ACM SIGCOMM workshop on Hot topics in software defined networking, pages 55-60. ACM, 2013.

[10] D. Kreutz, F. MV Ramos, PE Verissimo, C E. Rothenberg, S. Azodolmolky, and S. Uhlig. Software-defined networking: A comprehensive survey. proceedings of the IEEE, 103(1):14-76, 2015.

[11] K. Bakshi. Considerations for software defined networking ( $\operatorname{sdn})$ : Approaches and use cases. In Aerospace Conference, 2013 IEEE, pages 1-9. IEEE, 2013.

[12] A. Devlic, W. John, and P. Skoldstrom. A use-case based analysis of network management functions in the onf sdn model. In Software Defined Networking (EWSDN), 2012 European Workshop on, pages 85-90. IEEE, 2012.

[13] B. Lantz, B. Heller, and N. McKeown. A network in a laptop: rapid prototyping for software-defined networks. In Proceedings of the 9th ACM SIGCOMM Workshop on Hot Topics in Networks, page 19. ACM, 2010 . 